\title{
Ein modular aufgebautes Computerprogramm zur Ergebniswertberechnung von Radioimmunoassays und Proteinbindungstests 1 ),
}

\author{
Von W. Vogt, Burgi Popp') und M. KNEDEL \\ Klinisch-Chemiscbes Institut (Chefarzt: Priv. Doz. Dr. M. Knedel) am Städtischen Krankenbaus München-Harlacbing
}

(Eingegangen am 6. Dezember 1972/25. April 1973)

Ein modular aufgebautes Rechnerprogramm zur Ergebniswertberechnung von Radioimmunoassays und Proteinbindungstests wird beschrieben. Wegen der großen Bedeutung, die diese Bestimmungsverfahren zunehmend im Bereich der klinischen Chemie und der klinischen Forschung gewinnen, sahen wir unser Ziel darin, neben den selbstverständlichen Forderungen nach optimaler Genauigkeit und Sicherheit ein möglichst hohes $\mathrm{Ma}$ an Variabilität und Vielseitigkeit in der Anwendung zu erreichen und dabei eine einfache und voraussetzungslose Bedienung zu gewährleisten. Die Realisierung dieses Konzepts erfolgte über fünf von einander weitgehend unabhängige Untereinheiten.

Die bisher bekannt gewördenen mathematischen Auswerteverfahren wurden übersichtlich zusammengestellt und ausführlich diskutiert. Eine eigene Näherungsformel wird vorgeschlagen.

\section{A modillar constructed computer program for the calculation of the results of radioimmunoassays and protein il binding tests}

A modular computer program is described for the calculation of the results of radioimmunoassays and protein binding tests. In addition to the obvious need for optimal accuracy and reliability, and in view of the ever increasing importance of these analytical methods in clinical chemistry and research, we tried to design a program containing the greatest possible variability and versatility, which could be easily applied without preconditions. This concept was realized with the aid of five largely independent units.

The known mathematical calculation procedures are reviewed and discussed in detail. We have put forward our own approximation formula.

Die in dẹ meisten Laboratorien übliche, manuelle Ergebniswertberechnung von Radioimmunoassays und Proteinbindungstests ist zeitaufwendig und fehleranfällig. Das ist zum einen durch die hohe Meßwertanzahl, zum andern durch die rechnerische Aufbereitung der primären Meßergebnisse und das Ablesen der unbekannten Konzentrationen an grafischen Bezugskurven bedingt. Aus diesen Gründen wurden in den letzten Jahren bereits vereinzelt Rechner zur maschinellen Auswertung eingesetzt.

Die Programme sind entweder für Tischrechner (RoDBARD et al. (1)) mit ihren sehr begrenzten Möglichkeiten oder für Großrechner (Duddleson (2)) ausgelegt. Sie können somit nur bedingt die Aufgabenstellung des Routinelaboratoriums erfüllen.

Kleinrechner finden wegen ihrer unbestreitbaren Vorteile gegenüber Großcomputern in der Basisverarbeitung von Meßdaten mehr und mehr Eingang in die klinisch-chemischen Laboratorien.

Unser Ziel war deshalb, ein flexibles, praxisbezogenes Programmsystem für diese Maschinen zu entwickeln. Unsere Forderungen an ein solches Programm waren:

1. Hohe Auswertegenauigkeit durch ein entsprechendes Näherungsverfahren bei der Erstellung der Bezugskurve.

2. Vielseitigkeit in der Anwendung und ein hohes $\mathrm{Ma}$ an Flexibilität.

3. Einfache und voraussetzungslose Bedienbarkeit.

\section{Methodik}

\section{Gerät}

Das Programm wurde auf einer Varian $620 \mathrm{~L}$ mit $16 \mathrm{k}$ Kernspeicher erstellt. Als Peripheriegeräte waren ein optischer Lochstreifenleser und ein Blattschreiber (Teletype ASR 33) als Ein-Ausgabe-Einheit angeschlossen. Die radioaktiven Proben wurden entweder in einem Flüssigkeitsszintillationsspektrometer (Mark I, Fa. Nuclear-Chicago Comp., Des Plaines, Ill., USA) oder einem Gammaszintillationsspektrometer (Model 5375, Fa. Packard Instr. Comp., Downers Grove, Ill., USA) gezählt und die primären Meßwerte jeweils auf einer Teletype ASR 33 im Klartext als Protokoll und im ASCII-Code auf 8-Spur-Lochstreifen ausgegeben.

\section{Programmbeschreibung}

Das Programm ist modular aufgebaut. Zur Codierung wählten wir die problemorientierte, leicht erlernbare Sprache $\mathrm{BASIC}^{3}$ ). Kleinere Änderungen können so ohne programmtechnische Schwierigkeiten und ohne größere EDV-Kenntnisse vom Anwender selbst vorgenommen werden.

1) Arbeit im Rahmen des DV-Projekts 5.304 „Datenerfassung und Datenverarbeitung im Klinisch-chemischen Laboratorium" des Bundesministers für Forschung und Technologie.

2) EDV-Team des Universitätsklinikums München-Großhadern.

3) BASIC = Beginners All-Purpose Symbolic Instruction Code. 


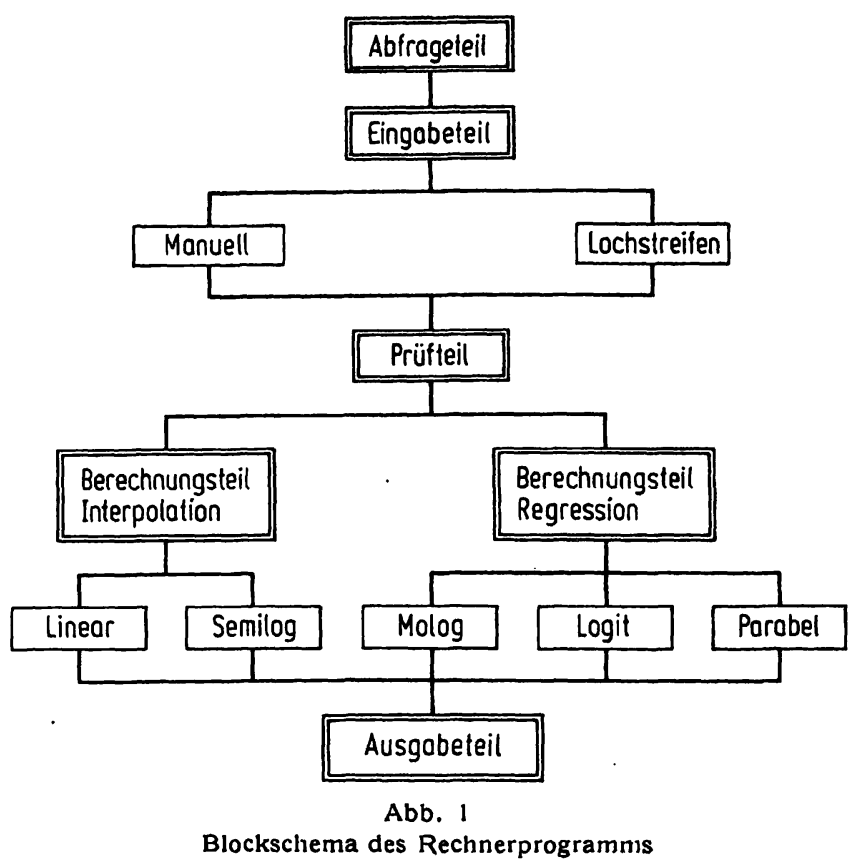

Die geforderte Flexibilität ist durch die Einteilung des Programms in 5 weitgehend voneinander unabhängige Blöcke gewährleistet (s. Abb. 1).

\section{Abfrageteil.}

Im Abfrageteil (s. Abb. 2) werden in einem einfachen Dialog die zum Programmablauf und zur Berechnung nötigen Steuerparameter manuell über den Blattschreiber eingegeben. Er wurde ausführlich angelegt, um möglichst vielen methodischen und apparativen Gegebenheiten gerecht werden zu können.

Mit nur geringen Änderungen im Programm kann der Dialog auf einem Datensichtschirm geführt werden, während die Datenausgabe weiterhin auf dem Blattschreiber oder besser noch einem Schnelldrucker erfolgt. Die Laufzeit des Programms kann dadurch erheblich verkürzt werden.

Wird das Programm nur in einem Auswertemodus betrieben, empfiehlt es sich, zur weiteren Zeitersparnis die Steuerparameter als feste Konstanten zu programmieren.

\section{Eingabeteil}

Die Standardkonzentrationen und die primären $\mathrm{Meß}$ werte können manuell oder über Lochstreifen je nach gewähltem Verfahren eingegeben werden (s. Abb. 3).

\section{Manuelle Eingabe}

Die. Konzentrationen der Standards sowie die Meßwerte von Standards und unbekannten Proben werden in einer definierten Folge abgefragt und über den Blattschreiber manuell eingegeben. Da die einzelnen Werte unter ihrer genauen Bezeichnung angefordert werden, erfolgt erst bei der Eingabe die endgültige Zuordnung zu den einzelnen Proben.

\section{Eingabe über Datenlochstreifen}

$\mathrm{Da}$ bei der Eingabe über Lochstreifen die Zuordnung der einzelnen Meßdaten zu den betreffenden. Proben lediglich über ihre Position innerhalb der Untersuchungsserie erfolgt, müssen die Zählgefäße bereits in der richtigen Reihenfolge in das Analysengerät gestellt werden. Sie werden in Gruppen zusammengefaßt. Die Reihenfolge der Gruppeneingabe ist:

1. Proben „Freies Antigen“4).

2. Proben „AG/AK-Komplex" ${ }^{\text {"4) }}$.

3. Proben gesamt $=$ „Freies Antigen $+\mathrm{AG} / \mathrm{AK}-\mathrm{Kom}-$ plex".

Die Reihenfolge innerhalb einer Gruppe ist ebenfalls festgelegt:

1. Leerwerte $=$ kein Antikörper, nur radioaktives Antigen,

2. Nullwerte $=$ kein stummes Antigen, nur radioaktives Antigen + Antikörpcr,

3. Standards und

4. unbekannte Proben.

Gleichzeitig mit der Eingabe werden die Meßwerte auf der Ausgabeeinheit protokolliert.

Das Einlesen der Meßwerte erfolgt über eine Unterroutine. Die zu jedem Meßwert gehörende SampleNummer des Analysengerätes wird gelesen und geprüft. Der auf den Meßwert folgende Wert wird überlesen (in unserem Fall gibt das Analysengerät gleichzeitig noch den Variationskoeffizienten der jeweiligen Messung an). Intern werden die Leerwerte auf Felder der Dimension (10), die übrigen Meßdaten auf Felder der Dimension $(120,3)$ abgespeichert. Insgesamt können so 120 Proben einschlięlich Standards bis zu 3-fach bestimmt und eingegeben werden.

\section{Prïfteil}

Im Anschluß an das Einlesen werden die Impulse/ Minute (cpm) für die quadratische Regression in die Quotienten $F / B_{\text {korr. }}$ und für die logarithmischen Regressionen in die Quotienten $B / B_{0}$ umgerechnet, wobei $F$ die Radioaktivität des freien Antigens, $B_{\text {korr. }}$ die des mit den Leerwerten korrigierten gebundenen Antigens, $B$ die des unkorrigierten gebundenen Antigens und $B_{0}$ die des gebundenen Antigens des Nullwertes bedeuten. Es folgt dann die Aussonderung von Ausreißern. Mehrfach bestimmte Werte, deren Abweichung vom Mittelwert größer ist als der angegebene Prozentsatz, werden eliminiert und die verbleibenden mit einer verkleinerten Schwelle nochmals überprüft. Falls eine Korrektur des Standard-Bezugspunktes nicht möglich ist, wird die Berechnung ganz abgebrochen.

\section{Berechnungsteil}

Berechnung der Bezugskurve

Die Berechnung der Bezugskurve erfolgt entweder durch Interpolation oder durch Regression. Die Auswertemöglichkeiten sind frei wählbar. Als Interpolationsverfahren stehen ein lineares und ein logarithmisches Verfahren zur Wahl. Für die Regressions-

\footnotetext{
4) Hier und im folgenden steht Antigen (AG) für den betreffenden Liganden und Antikörper ( $A K$ ) für das betreffende Bindungsprotein.
} 


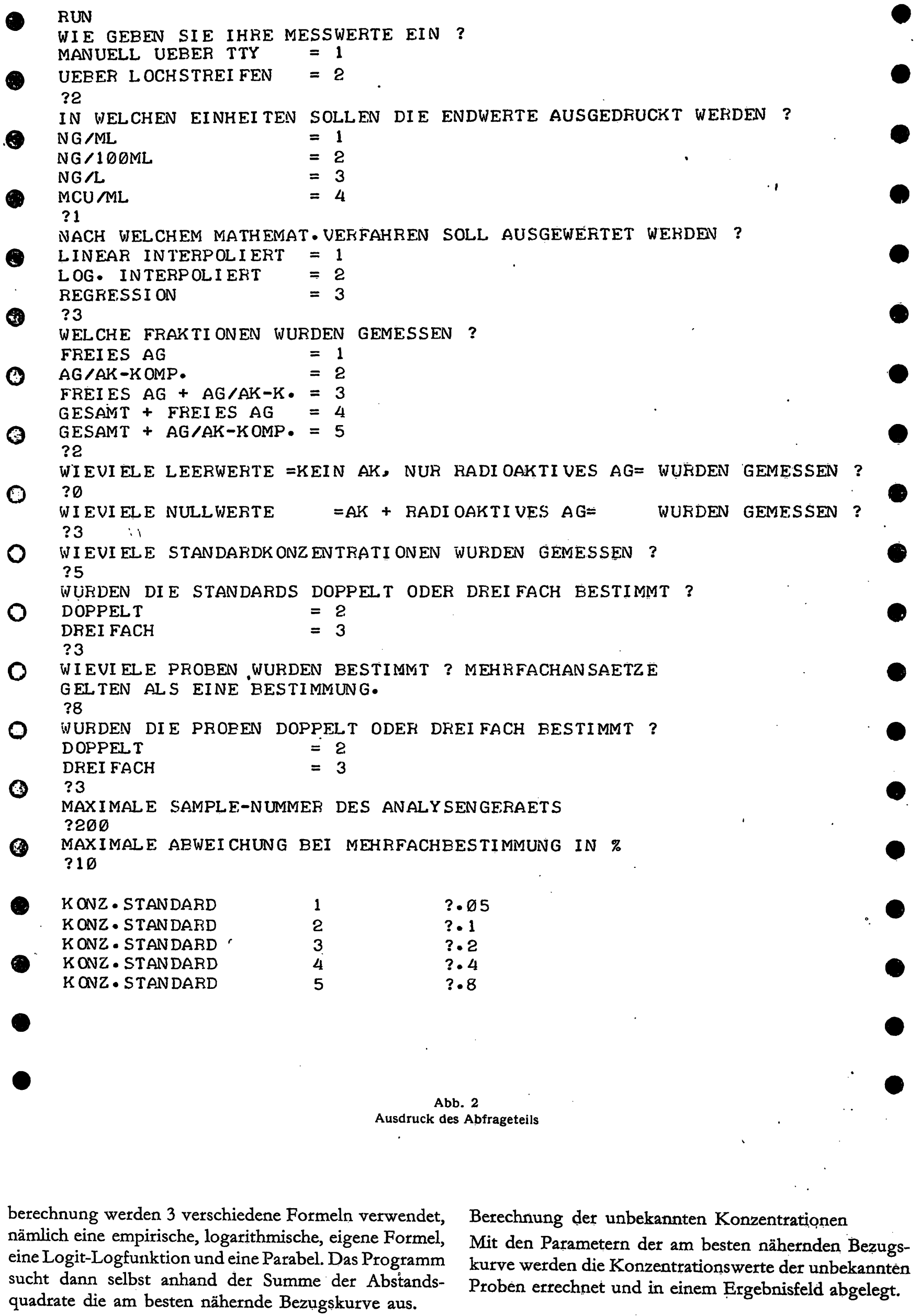


SEI TE 2

2)

Q NULLWEKT AG/AK-KOMF. 1

72779

NULLWEKT AG/AK-KOMH. 2

NULLWERT AG/AK-KOMP. 3

76822

77965

(2)

STANDARD AG/AK-KOMP - 1

53430

STANDARD AG/AK-KOMP. 1

53603

52032

37097

STANDARD AG/AK-KOMP. 2

38007

STANDARD AG/AK-KOMP. 2

- STANDARD AG/AK-KOMP.?

STANDAFD AG/AK-KOMP. 3

37920

242.65

STANDARD AG/AK-KOMP. 3

24513

24721

14430

14822

14834

8686

8390

STANDAFD AG/AK-KOMP. 5

9112

- STANDAKD AG/AK-KOMP. 5

81941

82552. 1

83242

69776

74915

73046

64314

65977

65543

63211

62772

62535

8.3662 .1

83452.1

81028

73046

77479

74355

66417

62379

$65456 \cdot 1$

(8)

-

60667

57410

592.57

0

0

(3)

$\mathbf{0}$

PFIOEE AG/AK-KONF.

PROEE AG/AK-KOMF.

PEOBE AG/AK-KOMP.

PFOEE AG/AK-KOMF.

PROBE AG/AK-KONP.

PAFAROL - FEGEESSI ON NICHT MOEGLICH

웅

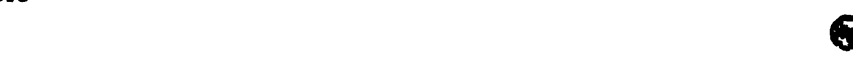

(1)

Abb. 3

Ausdruck des Eingabeteils

\section{Ausgabeteil}

Die Ausgabe erfolgt in Form eines Ergebnisjournals auf dem Blattschreiber (s. Abb. 4). Liegt der errechnete Wert außerhalb des Definitionsbereichs der Kurve, wird eine Fehlermeldung ausgegeben. Zusätzlich werden bei Wahl der Regressionen noch das Verfahren, das vom Rechner als am besten nähernd exkannt wurde, der mittlere Variationskoeffizient und bei den logarithmischen Verfahren der Korrelationskoeffizient der Bezugskurve angegeben.

\section{Feblermeldungen}

Besonderer Wert wurde auf die Fehlerprüfroutinen und entsprechende Fehlermeldungen in Klartext gelegt. 
SEITE 3

KLINI SCH-CHEMI SCHES INSTI TUT

STAEDT.KRANKENHAUS HARLACHING

B.EREI CH ENDOKRINOL OGI E

UND STOFFWECHSEL.

C.

AUSWERTUNG FADI OIMMUNOASSAYS, REGRESSI ON MOLOG, R=-.998967, $M V K=1.17462$

LAUF.•NR・

-

$\begin{array}{ll}\text { PROEE } & 1 \\ \text { PROEE } 1 \\ \text { PROBE } 1 \\ \text { PROBE } 2 \\ \text { PROBE } 2 \\ \text { PROBE } 2 \\ \text { PROBE } & 3 \\ \text { PROBE } & 3 \\ \text { PROEE } & 3 \\ \text { PROBE } & 4 \\ \text { PROBE } & 4 \\ \text { PROEE } & 4 \\ \text { PROBE } 5 \\ \text { PROBE } & 5 \\ \text { PROBE } & 5 \\ \text { PROBE } & 6 \\ \text { PROBE } 6 \\ \text { PROBE } 6 \\ \text { PROBE } 7 \\ \text { PROBE } 7 \\ \text { PROBE } 7 \\ \text { PROEE } 8 \\ \text { PROEE } 8 \\ \text { PROBE } 8 \\ \text { READY }\end{array}$

NG/ML

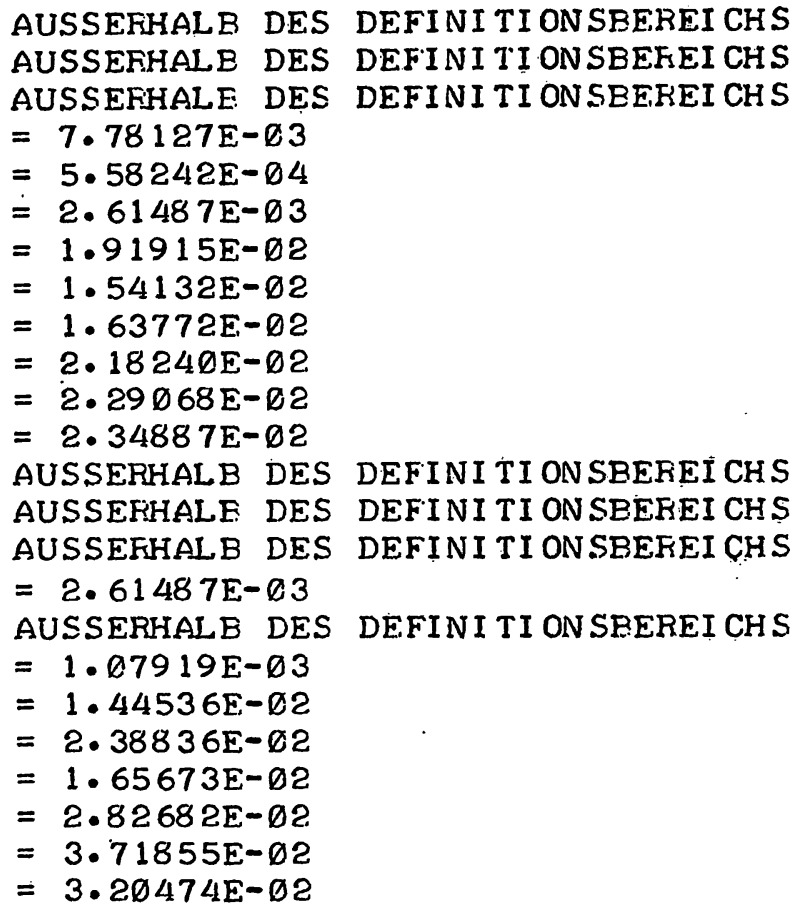

2

Abb. 4

Ausdruck des Ausgabeteils

Werden Fehler erkannt, die vom Programm nicht korrigiert werden können, so wird die Auswertung sofort abgebrochen. Sind die Fehler korrigierbar, wird nach der Ausgabe einer entsprechenden Fehlermeldung der Programmlauf fortgesetzt.

\section{Diskussion}

Auswerteverfahren

Die Beschreibung der Bezugskurven von Radioimmunoassays und Proteinbindungstests (CPBA) durch einfache mathematische Gleichungen ist nur bedingt möglich. Die Näherung führt nur in begrenzten Kurvenabschnitten $\mathrm{zu}$ guten Resultaten. Des weiteren wurden von RODBARD et al. (3) und TäLJEDAL und Word (4) gerade bei dem verbreitetsten Verfahren, nämlich der Logit-log-Transformation, statistisch begründete Bedenken geäußert, da die zwangsläufige, formelbedingte, mathematische Gewichtung der einzelnen Bezugspunkte nicht mit der tatsächlichen, experimentell begründeten übereinstimmt. Deshalb ist die
Berechnung der Endwerte anhand solcher nichtlinearer Bezugskurven bisher nicht voll befriedigend und die Richtigkeit der so erhaltenen Ergebnisse unterschiedlich.

Eine größere Richtigkeit kann meist nur mit unverhältnismäßig hohem mathematischem Aufwand erkauft werden. Aus praktischen Erwägungen muß deshalb ein befriedigender Mittelweg zwischen der Alternative - Richtigkeit des Endwerts und Einfachheit seiner Errechnung - gefunden werden. $\mathrm{Da}$ viele Lösungsmöglichkeiten denkbar sind, ist eine Anzahl von Vorschlägen zur mathematischen Bezugskurvennäherung von Radioimmunoassays und Proteinbindungstests (CPBA) bekannt geworden.

In Tabelle 1 haben wir die Verfahren und Formeln nach der Literatur zusammengestellt.

\section{Heuristische Regressionsverfabren}

Die Anwendung von Polynomen ist in der Statistik allgemein üblich und gut bekannt. Sollen Bezugskurven oder biologische Kurven genähert werden, 
Tab. 1

usammenstellung der bisher bekannt gewordenen Verfahren zur mathematischen Năherung von Radioimmunoassay- und Proteinbindungstest (CPBA)-Bezugskurven nach der Literatur

labei bedeuten:

- In der Spalte "Gleichung" $y$ und $x$ die beiden Variablen, z eine Hilfsvariable und $a, b$ und c die entsprechenden Paraneter der Funktion.

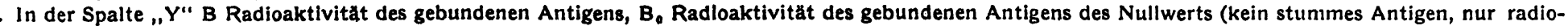
aktiv markiertes Antigen und Antikörper im Ansatz), F Radioaktivität des freien Antigens und T Gesamt-Radioaktivität des Ansatzes vor der Trennung. - In der Spalte „X" C die Konzentration der nachzuweisenden Substanz im Ansatz und C* die Menge der radioaktiv markierten, zugegebenen Substanz.

$\begin{array}{llcc}\text { Nummer Näherungsverfahren } & \text { Gleichung } & \text { Literaturstelle } \\ \text { für Klein- } \\ \text { rechner }\end{array}$

1. Heuristische Verfahren

1.1. Lineare Regressionsverfahren

1.1.1. Regression mit halblogarith-

mischem Polynom 1. Ordnung

$y=a+b \log x$

$x=a+b \log y+c \log ^{2} y$

1.1.2. Regression mit halblogarith-
mischem Polynom 2. Ordnung

1.1.3. Regression mit doppelt loga-

rithmischem Polynom 1. Ordnung

(Exponentialfunktion I)

1.1.4. Regression mit Polynom 2. Ordnung

1.1.5. Regression mit Polynom 3. Ordnung

1.1.6. Arcus-Sinus-Transformation

1.1.7. Probit-Transformation

1.1.8. Modifizierte, logarithmische

Regression (Molog)

$\log y=a+b \log x$
$y=a+b x+c x^{2}$
$y=a+b x+c x^{2}+d x^{3}$
$\sin ^{-1} y=a+b \log x$
Probit $(y)=a+b \log x$
$z=a+b \log x$, wobei
$z\left\{\begin{array}{l}\log y \text { für } y \leq 50 \\ \log \left(\frac{2500}{100-y}\right) \text { für } y>50\end{array}\right.$

1.2. Nichtlineare Regressionsverfahren

1.2.1. Exponentialfunktion II $\quad y=a e^{b x}+c$

1.3. Interpolationsverfahren

1.3.1. Lineare Interpolation $y=a_{1}+b_{1} x$

1.3.2. Logarithmische Interpolation $\log y=a_{i}+b_{i} x$

2. Verfahren, denen theoretische Uberlegungen zugrunde liegen

2.1. Lineare Regressionsverfahren

2.1.1. Hyperbolische Regression 1

2.1.2. Hyperbolische Regression II

$\frac{1}{y}=a x+1$

$\frac{1}{y}=b x+c$

2.1.3. Logit-Transformation

$\log \left(\frac{y}{100-y}\right)=a+b \log x$

2.2. Nichtlineare Regressionsverfahren

2.2.1. Hyperbolische Regression $111 \quad \frac{1}{y+a}=b x+c$

2.2.2. Verallgemeinerte Logit-

Transformation

$y=\frac{a}{x^{c}+b}$

2.2.3. Theoretisch begründete Hyperbel

2.3.1. Hyperbolische Regression IV

$$
y^{2}+y\left(1+K x-K A b_{0}\right)-K A b_{0}=0
$$

$y=\frac{c}{x+c}$

$\frac{y}{1-y}=a+b y x$
EKINS et al. (1968) (5)

MORGAN et al. (1967) (15)

BROWN et al. (1970) (7)

YALOW \& BERSON (1968) (6)

TÄLJEDAL \& WOLD (1970) (4)

TÄLJEDAL \& WOLD (1970) (4)

VIVIAN \& LABELLA (1971) (8)

FINNEY (1964) (16)

FINNEY (1964) (16)

Vogt et al. (1972)

TÄLJEDAL \& WOLD (1970) (4)

allgemein bekannt

allgemein bekannt

Hales \& RANDle (1963) (10)

MURPHY (1967) (11)

RODBARD et al. (1968, 1969)

$(1,3)$

TÄLJEDAL \& WOLD (1970) (4)

RODBARD et al. $(1968,1969)$

$(1,3)$, zit. nach TÄLJEDAL \&

WOLD (1970)

BERSON \& YALOW (1968) (17)

RODBARD et al. (1969) (1)

RODBARD et al. (1969) (1)

$\begin{array}{lll}+ & B / F & C \\ + & B / B_{0} & C\end{array}$

$+$

$100 \mathrm{~B} / \mathrm{T}$

C

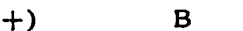

$+\quad B / B_{0} \quad C$

$\begin{array}{ccc}- & B / B_{0} & C \\ + & 100 \mathrm{~B} / \mathrm{B}_{0} & \mathrm{C}\end{array}$
2.3. Grafisch unterstützte Verfahren

B

B oder $F$

B oder $\mathbf{F}$

$+$

B/B。

$B / B_{0}$

$+$

$+100 \mathrm{~B} / \mathrm{B}_{0}$

- B

C

$-\quad B / B_{0}$

C

2.3.2. SCATCHARD-Plot empfiehlt sich häufig ein logarithmischer Maßstab. Die rechnerische Handhabung von Polynomen 1. Grades ist naturgemäß besonders einfach und auch auf Tischrechnern leicht zu realisieren. ExINS et al. (5) sowie YALOW und BERson (6) konnten damit ihre Bezugskurven über einen größeren Teilbereich gut nähern. BRowN (7) wandte mit gutem Exfolg halblogarithmische, quadratische Polynome an. Näherungsversuche von TäLJEDAL und Word (4) mit Polynomen 2. und 3. Ordnung erwiesen sich dagegen nicht als nutzbringend. Sie setzen allerdings als Ordinatenwerte direkt die ge- messene Radioaktivität ein. Benutzt man jedoch $\mathrm{F} / \mathrm{B}$ als unabhängige Variable, so erhält man nach unseren Erfahrungen sehr gute Ergebnisse. In dieser Form haben wir diese quadratische Gleichung auch in unser Programm aufgenommen.

Vivian und Labella (8) wandten eine Arcus-SinusTransformation zur Linearisierung ihrer Standardkurven an. Rodbard et al. $(1,3)$ stellen dieses Verfahren in der statistischen Wertigkeit mit der Probit und Logit-log-Transformation gleich, verwenden aber selbst das Logit-Verfahren. Wir haben auf empirischem 
Weg eine zusammengesetzte Exponentialfunktion gefunden (VoGT et al. 9), die ebenfalls eine gute Näherung erlaubt:

$$
\operatorname{molog}(y)=\left\{\begin{array}{l}
\log y \text { für } y \leqq 50 \\
\log \frac{2500}{100-y} \text { für } y>50 .
\end{array}\right.
$$

Wir konnten mit Hilfe dieser Formel in vielen Fällen, in denen eine Logit-log-Transformation nicht zum Ziel führte, die Kurve gut nähern.

\section{Interpolationsverfabren}

Lineare und logarithmische Interpolation sind zur Näherung mathematisch nur schwierig oder ungenau beschreibbarer Kurven allgemein üblich. Thre Durchführung ist einfach, die Richtigkeit der Näherung ist jedoch fragwürdig und hängt von der Anzahl und Genauigkeit der Bezugspunkte und der Kurvenform ab.

Trotz dieser augenscheinlichen Mängel haben wir die Interpolationsverfahren in unserem Programmsystem vorgesehen. Es sollte auch dann noch eine Auswertung von Meßdaten möglich sein, wenn die angebotenen Regressionsverfahren versagen und durch Variation der Nachweismethode keine weitere Optimierung mehr erreicht werden kann. Die Ordinate entspricht dann der gemessenen Radioaktivität bzw. deren Logarithmus, die Abszisse der Konzentration.

Verfabren, denen theoretische Überlegungen zugrunde liegen Nahezu alle diese Verfahren lassen sich auf die von HaLes und RANDle (10) angegebene theoretische Beziehung zurückführen, wonach

$$
\frac{c_{0}}{c_{1}}=\frac{1}{i_{0}} \cdot i+1
$$

Dabei ist $c_{i}$ die Radioaktivität des Antigen-Antikörper-Komplexes, wenn die Konzentration des nicht markierten Antigens $i$ ist, $c_{0}$ die Radioaktivität für $\mathrm{i}=0$ und $\mathrm{i}_{0}$ die Konzentration des markierten Antigens.

Es liegt also theoretisch eine hyperbolische Bezugskurve der Form

$$
\frac{1}{y}=a x+1
$$

für die relative Aktivität des gebundenen Antigens vor.

$\mathrm{Da}$ diese Beziehung mathematisch sehr einfach ist, wird sie von RODbard et al. (1) für ein grafisches Auswerteverfahren angegeben. Sie gilt jedoch nur im Sättigungsbereich exakt. Sie wurde daher von MURPHY (11) durch Einführung eines zusätzlichen Parameters verallgemeinert.

$$
\frac{1}{y}=a x+b
$$

In dieser Form wurde die Gleichung (3) von WomBACHER und KörBer (12) mit Erfolg verwendet und theoretisch begründet. Noch flexibler ist die hyperbolische Regression in der von TäLJEDAL und WoLD (4) vorgeschlagenen Form. Sie ist nach Aussage der beiden Autoren hervorragend geeignet, läßt sich jedoch nicht explicit, sondern nur durch iterative Rechenverfahren lösen, da die der Regression zugrunde liegende Gleichung nicht linear in den Parametern ist. Somit kann diese Methode nur für Großrechner geeignet sein. Durch Umrechnen erhält man aus Gleichung (2) eine Gleichung (4) der Form

$$
\operatorname{logit} y=a-\log x
$$

für die prozentuale gebundene Radibaktivität. Sie gilt wiederum nur im Sättigungsbereich der Kurve, wenn also die Menge an gebundenem Antigen (markiert plus unmarkiert) konstant ist. $\mathrm{Da}$ diese jedoch ungefähr dem Logarithmus der Konzentration proportional ist, gilt allgemein

$$
\operatorname{logit} y=a+b \log x
$$

(Rodbard et al. 1).

Diese Logit-Transformation ist wohl das augenblicklich am häufigsten benutzte Verfahren. Ein großer Teil unserer Bezugskurven war mit seiner Hilfe gut bis sehr gut linearisierbar. $\mathrm{Da}$ es sich auch wegen der Einfachheit der Handhabung für eine Rechnerauswertung eignet, haben wir es in unser Programm aufgenommen. Zudem muß lediglich die Radioaktivität des gebundenen Antigens gemessen werden.

Eine Verallgemeinerung der Logit-Regression stellt die Gleichung

$$
y=\frac{a}{x^{c}+b}
$$

dar. Da diese Regressionsgleichung nicht linearisierbar ist, läßt sie sich nicht auf rechnerisch einfachem Wege lösen. Zudem bringt sie nach TÄLJEDAL und WOLD (4) keine Vorteile gegenüber den anderen Verfahren.

BERSON und YALOW (13) haben schon 1959 gezeigt, daß für eine univalente, homogene Antigen-AntikörperReaktion folgende Beziehung gilt:

$$
\mathrm{B} / \mathrm{F}=\mathrm{K}\left(\mathrm{Ab}_{0}-\mathrm{B}\right) \text {. }
$$

Dabei entspricht $B$ der Menge an gebundenem, $F$ der an freiem Antigen, $A b_{0}$ der Konzentration der Bindungsstellen des Antikörpers (= Bindungskapazität des Antikörpers) in der Lösung und $\mathrm{K}$ der Gleichgewịchtskonstanten $=$ Affinitätskonstanten. Daraus ergibt sich eine hyperbolische Gleichung

$(\mathrm{B} / \mathrm{F})^{2}+\mathrm{B} / \mathrm{F}\left(1+\mathrm{KC}-\mathrm{KAb_{0 }}\right)-\mathrm{KAb_{0 }}=0 .(\mathrm{Gl} .8)$

(BROwN et al. (7)). Eine Regression ist aufgrund dieser Beziehung nicht ohne weiteres möglich und wurde deshalb bisher unseres Wissens noch nicht angewandt.

RODBARD et al. (1) verwenden diese Gleichung nach Umrechnen in die Quotienten B/T für einen "SCATCHARD-Plot" (14). Dies stellt jedoch eine rein grafische Lösung dar.

\section{Ausreißersuche}

Zur Erkennung und Elimination von Ausreißern bewährte sich in unserem Laboratorium folgende Prüfroutine. Bei Dreifachbestimmungen werden die Meßwerte gemittelt und gegebenenfalls der außerbalb des Prüffensters liegende Wert eliminiert. Die Breite dieses 
Fensters ist frei wählbar und sollte etwa das 2 bis 2,3-fache der Standardabweichung betragen (WAGNER (15)). Die grundsätzliche Entscheidung, ob es sich dabei um einen relativen oder absoluten Fehler handelt, ist nur bedingt möglich. Bei der parabolischen Regression schien es sich nach unseren Erfahrungen vorwiegend um einen relativen Fehler zu handeln, bei den logarithmischen Kurven eher um einen absoluten. $\mathrm{Da}$ wir uns aus technischen Gründen festlegen mußten, entscheiden wir uns wegen der leichteren Handhabbarkeit für eine prozentuale Angabe der Prüffensterbreite. Nach der Eliminierung des Ausreißers werden die beiden verbleibenden Werte nochmals gemittelt und in einem um ein Drittel reduzierten Fenster geprüft. Liegen die Werte wiederum außerhalb des gewählten Bereichs, wird eine Fehlermeldung ausgegeben und das Programm abgebrochen. Nach visueller Uberprüfung der Meßwerte kann die Bezugskurve mit vergrößertem Prüffenster oder unter Auslassen des betreffenden Standards nochmals eingegeben werden.

\section{Wahl der Programmsprache}

Die eingangs kurz skizzierten Vorzüge von BASIC, die uns veranlaßten, das Programm primär in dieser Sprache zu codieren, verleiten möglicherweise den in der Datenverarbeitung weniger Erfahrenen zu dem Urteil, es handle sich um eine für solche Probleme besonders geeignete Sprache. Wir weisen deshalb abschließend auf einige Nachteile hin. So erfolgt die Compilierung während des Programms. Das bewirkt eine erhöhte Laufzeit und erhöhten Kernspeicherbedarf. Zum anderen sind die Möglichkeiten in der Verarbeitung mehrdimensionaler Felder begrenzt, so daß der Transfer in Unterroutinen nur bedingt möglich ist. Wir codieren deshalb für die Anwendung in unserem Labor das Programm augenblicklich in FORTAN IV um.

Wissenschaftlich an den Einzelheiten des EDV-Programms Interessierte erhalten eingehende Auskunft bei den Verfassern.

\section{Literatur}

1. Rodbard, D., Bridson, W. \& RaYrord, P. L. (1969), J. Lab. Clin. Med. 74, 770-781. - 2. Duddleson, W. G., Midhley, A. R. \& Niswender, G. D. (1972), Comp. Biomed. Res. 5, 205-217. - 3. Rodbard, D., Raypord, P. L., Cooper, J. A. \& Ross, G. T. (1968), J. Clin. Endocrinol. 28, 1412-1418. 4. TÄLJEDAL, I. B. \& WOLD, S. (1970), Biochem. J. 119, 139-143. 5. Ekins, R. P., Newman, G. B. \& O'Riordan, J. L. H. (1968), in: Radioisotopes in Medicine: In vitro studies. Theoretical Aspects of Saturation and Radioimmunoassay (Hrsg. Hayes, R. L., Goswitz, F. A. \& Murphy, B. E. P.) 1968 ed., p. 59, Oak Ridge, Tennessee (USA). Zit. nach 1. c. (1). - 6. YALOW, R. S. \& BERson, S. A. (1968), in l. c. (5), Zit. nach 1. c. (1). - 7. Brown, G. M., Boshans, R. L. \& Schalch, Don S. (1970), Comp. Biomed. Res. 3, 212-217. - 8. Vivian, ST. R. \& Labelia, F. S. (1971), J.
Clin. Endocrinol. 33, 225-233. - 9. Vogt, W., Popp, B. \& KNEDEL, M., in Vorbereitung. - 10. Hales, C. N. \& RANDLE, P. J. (1963), Biochem. J. 88, 137-146. - 11. Murphy, B. E. P. (1967), J. Clin. Endocrinol. 27, 973-990. - 12. WombaCHER, H. \& KöRBER, F. (1972), diese Z. 10, 260-266. - 13. Berson, S. A. \& Yalow, R. S. (1959), Zit. nach l. c. (1) und (17). 14. Scatcilard, G. (1949), Ann. New York Acad. Sc. 51, 660. Zit. nach 1. c. (1). - 15. WeBer, W. E. (1972), EDV Med. Biol. 1, 23-32. - 16. Morgan, C. R., Shardigg, B. S. \& Fisher, D. D. (1967), Diabetes 16, 734. Zit. nach l. c. (4). - 17. FINNEY, D. J. (1968), Statistical Methods in Biological Assay. Ed. 2. Charles Griffin \& Co. Ltd., London. Zit. nach 1. c. (3). - 18. Berson, S. A. \& Yalow, R. S. (1968), Clin. Chim. Acta 22, $51-69$.
Priv.-Doz. Dr. M. Knedel Städt. Krankenhaus München-Harlaching Klin.-chem. Institut 8000 München 90 Sanatoriumsplatz 2 